\title{
The use of tranexamic acid in microsurgery-is it safe?
}

\author{
Alessia M. Lardi ${ }^{1,2,3,4}$, Kathrin Dreier ${ }^{4}$, Klaus Junge ${ }^{5}$, Jian Farhadi ${ }^{1,2,3,4}$ \\ ${ }^{1}$ Breast Center Zürich, Zürich, Switzerland; ${ }^{2}$ Department of Plastic, Reconstructive, Aesthetic and Hand Surgery, University Hospital Basel, Basel, \\ Switzerland; ${ }^{3}$ Plastic Surgery Group (by Prof. Farhadi), Zürich, Switzerland; ${ }^{4}$ Clinic Pyramide at the Lake, Zürich, Switzerland; ${ }^{5}$ Premier Research, \\ Darmstadt, Germany \\ Contributions: (I) Conception and design: AM Lardi, J Farhadi, K Junge; (II) Administrative support: AM Lardi; (III) Provision of study materials or \\ patients: AM Lardi, J Farhadi; (IV) Collection and assembly of data: AM Lardi, K Dreier; (V) Data analysis and interpretation: AM Lardi, K Junge; (VI) \\ Manuscript writing: All authors; (VII) Final approval of manuscript: All authors. \\ Correspondence to: Alessia M. Lardi, MD. Breast Center Zürich, Zürich, Switzerland. Email: a.lardi@brust-zentrum.ch.
}

\begin{abstract}
Background: An appropriate and adequate blood flow and oxygen delivery is paramount to free flap viability and success. The perioperative use of tranexamic acid (TXA) is associated with less risk for blood loss and blood transfusion in trauma, gynaecology, ear nose and throat (ENT) and orthopaedic surgery. As an antifibrinolytic drug, TXA has generally been avoided in microsurgery. The aim of this study is to evaluate the safety and benefit of using TXA in microsurgery.

Methods: We performed a retrospective single centre cohort study at the Pyramid Clinic, Zurich, Switzerland, including 98 free tissue transfers for breast reconstruction from 2011 to 2013. According to the estimated blood loss, up to $3 \mathrm{~g}$ TXA were administered intravenously in 63 free flaps perioperatively.

Results: No thrombosis $(0 \%)$ of micro-anastomosis and 5 haematomas $(10.0 \%)$ occurred after administration of TXA. In the control group, 1 thrombosis (3.0\%) of a flap-vein and 6 haematomas $(18.2 \%)$ occurred. Blood loss was significant lower $(\mathrm{P}<0.001)$ after administration of TXA.

Conclusions: In this study, administration of TXA did not increase thrombosis in free tissue transfer and showed a reduced haematoma rate and significant reduction of blood loss. TXA is supposed to be safe and a reasonable adjunct for patients with anaemia and a higher intraoperative or postoperative blood loss.
\end{abstract}

Keywords: Tranexamic acid (TXA); microsurgery; free tissue transfer; breast reconstruction

Submitted Mar 18, 2018. Accepted for publication Mar 19, 2018.

doi: $10.21037 /$ gs.2018.03.10

View this article at: http://dx.doi.org/10.21037/gs.2018.03.10

\section{Introduction}

Free tissue transfer for breast reconstruction rarely involves significant blood loss $(1,2)$. However, our patients are generally cancer patients and do present with pre-existing anaemia related to their cancer or its treatment $(3,4)$. An appropriate and adequate blood flow and oxygen delivery is paramount to free flap viability and success (5). The perioperative use of tranexamic acid (TXA) is associated with less risk for blood loss and transfusion in trauma, gynaecology, ear nose and throat (ENT) and orthopaedic surgery (6-9). In a meta-analysis of surgical trials including
10,488 patients, the risk for transfusion was reduced by $38 \%$ in the TXA group, with no significant differences in mortality or thrombotic events (10). As an antifibrinolytic drug, TXA has generally been avoided in microsurgery. In literature, there is an inconsistent evidence for increased risk for thromboembolic complications $(9,11)$. Also, at present still no consensus has been established on treatment guidelines in terms of ideal antithrombotic agents, timing, and dosage in reconstructive microsurgery (12-14). Lowmolecular heparin in a recent literature review Brinkman et al. was recommended as monotherapy, as this seems to be as effective as acetylsalicylic acid, and has the additional 
advantage to prevent systemic thromboembolic events, and unlike acetylsalicylic acid does not increase the risk of gastrointestinal bleeding (12).

The aim of this study is to evaluate the safety and benefit of using TXA in microsurgery.

\section{Methods}

We performed a retrospective single centre cohort study from October 2011 until October 2013, including free tissue transfer for immediate breast reconstruction. This study represents a single-surgeon experience. The procedures were performed with a uniform protocol: low-molecular heparin, Enoxaparine, (Clexane ${ }^{\circledR}$, SanofiAventi) was administered in prophylactic dosage (40 mg s.c.) starting 12 hours before procedure and once daily until discharge as antithrombotic monotherapy. Neither intraoperative heparin nor postoperative acetylsalicylic acid was administered (12-14). Experienced senior breast surgeons performed the mastectomies via sharp dissection. No tumescent solution was used.

During period 1 (first 12 months), no TXA was administered. During period 2 (second 12 months), up to $3 \mathrm{~g}$ TXA were administered intravenously according to the estimated blood loss, both intraoperatively and/or postoperatively. The intraoperative blood loss was estimated on the amount of blood in the suction container and by the senior author evaluating swaps and blood loss on table twice, once after mastectomy was finished, and once after anastomosis was done. If intraoperative blood loss was estimated $>100 \mathrm{~mL}$ from last measurement, $1 \mathrm{~g}$ TXA was administered. The postoperative blood loss during first 24 hours was estimated from drain output. If drain output was fresh blood $>200 \mathrm{~mL}$ per hour another dosage of $1 \mathrm{~g}$ TXA was given till 24 hours after procedure.

Blood transfusions were administered if $\mathrm{Hb}<8 \mathrm{~g} / \mathrm{L}$ or if patients became symptomatic for anaemia. Patients with a known tendency for thromboembolic complications and thromboembolic events in their personal history were excluded. We also excluded delayed breast reconstructions, because of low number and to gain uniform procedure characteristics.

\section{Data collection and analysis}

Patient demographic data including age, body mass index, diabetes, current tobacco use, chemotherapy (neoadjuvant or adjuvant) and radiotherapy, as well as procedure specific parameters (type of flap performed, flap weight, ischemia time, intraoperative problems) were collected from hospital records.

Postoperative complications [haematoma, thrombosis, flap loss, blood transfusion, deep venous thrombosis (DVT), pulmonary embolism (PE)] were recorded, as well as intraoperative and postoperative blood loss during first 24 hours.

The two-groups $t$-test was used for continuous data, the Chi-square test for categorical data and the Fisher test for binary data. Exact confidence intervals for rates were calculated assuming a binomial distribution. The impact of risk factors on the occurrence of haematoma was analysed using a univariate logistic model. A value of $\mathrm{P}<0.05$ was considered significant.

Two patients contributed data from two different procedures, once with use of TXA and once without TXA. The data of the two procedures was treated as independent observations in the analysis of complications. For the analysis of risk factors, only the data from the procedure without use of TXA was used. For the analysis of blood loss an ANOVA with factors for treatment, flap type and the corresponding interaction was used.

\section{Results}

Over the 2-year study period, the senior author performed 100 free flaps for immediate breast reconstruction on 85 patients. We excluded two patients/free flaps for DVT in their medical history. Ninety-eight free flaps in 83 patients were included. In the treatment group, 50 patients and 63 free flaps were included, 33 patients and 35 free flaps in the control group. See demographic data in Table 1.

In total, 98 mastectomies were performed, of which 82 for oncological reasons and 16 for risk-reduction. In the study population, 48 deep inferior epigastric perforator (DIEP) flaps, 10 profunda artery perforator (PAP) flaps, 25 transverse myocutaneous gracilis (TMG) flaps and 15 superior gluteal artery (S-GAP) flaps were performed. Flap characteristics are summarised in Table 2.

Revision surgery was performed in 21 cases $(21.4 \%)$, of which 19 were successful without flap loss. Eleven flaps $(11.2 \%)$ were revised due to haematoma at the flap side. In three cases a kinking of the pedicle was found. One patient was brought back to theatres due to hypothermia of the flap, with no cause found in revision surgery. Three 
Table 1 Patient demographics and clinical characteristics

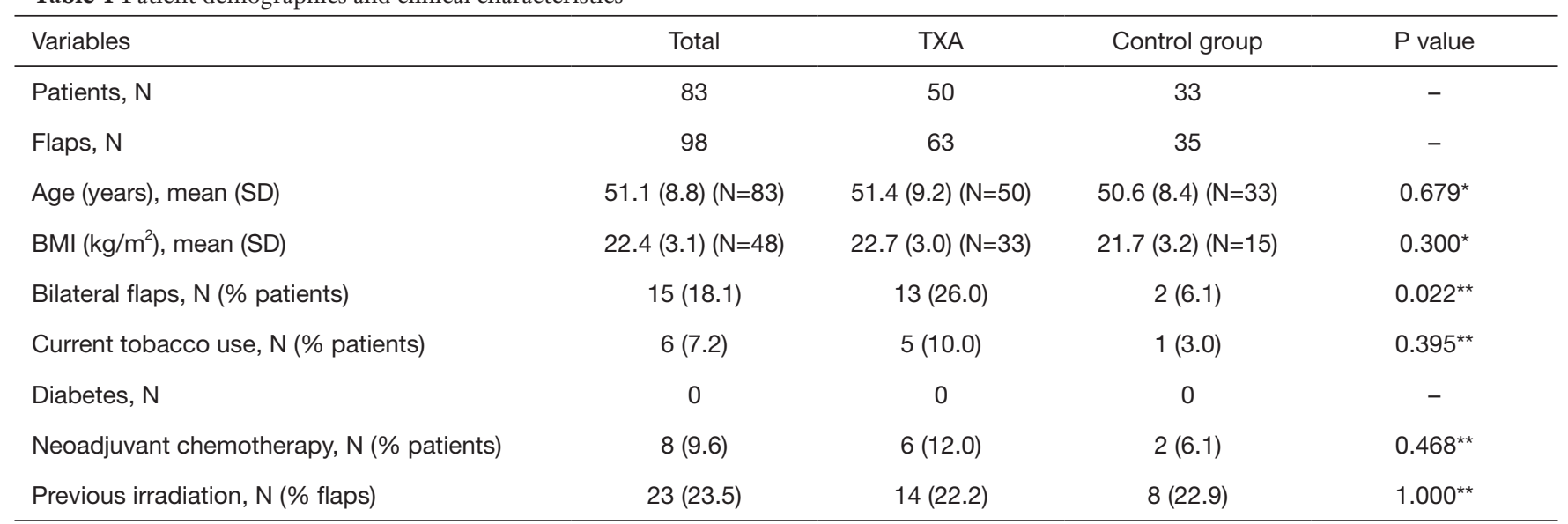

*, Student's t-test; ${ }^{* *}$, Fisher test. TXA, tranexamic acid.

Table 2 Flap characteristics

\begin{tabular}{lcc}
\hline Characteristics & TXA (N=63) & $\begin{array}{c}\text { Control group } \\
(\mathrm{N}=35)\end{array}$ \\
\hline Flap type, N (\%) & $31(49.2)$ & $17(48.6)$ \\
DIEP & $7(11.1)$ & $3(8.6)$ \\
PAP & $12(19.0)$ & $13(37.1)$ \\
TMG & $13(20.6)$ & $2(5.7)$ \\
S-GAP & $405[151]$ & $361[172]$ \\
Flap weight, mean [SD] (g) & $65.2(26.4)$ & $57.9(16.2)$ \\
Ischemia time, mean (SD) (min) & DIEP, deep inferior epigastric perforator; PAP, profunda artery \\
perforator; TMG, transverse myocutaneous gracilis; S-GAP, \\
superior gluteal artery; TXA, tranexamic acid.
\end{tabular}

flaps showed venous congestion, two because of inadequate venous outflow and no thrombosis was found. In these two cases an additional venous drainage resolved the impairment. In one case (DIEP) of venous congestion a thrombosis of the micro-anastomosis of the vein caused the clinical impairment, the thrombosis was removed and the venous anastomosis redone. In one free flap (S-GAP) the micro-anastomosis of the artery was redone for technical problems.

Flap loss occurred in two cases (2\%). In these two flaps (one S-GAP, one DIEP) the perforator was too small and insufficient for flap supply and flaps were lost.

Table 3 shows distribution of complications for TXA and control group.

\section{Discussion}

Only one thrombosis of a micro-anastomosis of a vein occurred in the study population (control group). In this study, the administration of TXA did not increase the risk of thrombosis. Haematoma rate $(\mathrm{P}=0.332)$ was reduced in the Tranexamic group. However, in this study, haematoma rate was higher compared to the literature $(1,2,15)$. In the senior author's practise, the decision for revision surgery is taken early before a developing haematoma may impair flap survival. In all haematoma revisions no real source was found. Evaluating the risk factors for haematoma in this study, not only the avoidance of the use of TXA $(\mathrm{P}=0.398)$, but also neoadjuvant chemotherapy $(\mathrm{P}=0.333)$, smoking $(\mathrm{P}=0.165)$ and higher age $(\mathrm{P}=0.035)$ showed to be risk factors for the occurrence of haematoma (Table 4).

Intraoperative and blood loss during the first 24 hours was reduced significantly $(\mathrm{P}<0.001)$ with the use of TXA. Only one patient in the study group needed a blood transfusion (TXA group). The patients in the control group, although with higher blood loss, did not need blood transfusion.

TXA in this study did not increase thrombosis and was able to reduce bleeding. This creates a new option in pharmaceutical treatment of surgical bleeding in free flap surgery, without putting at risk microsurgical anastomosis. Nevertheless, in our opinion, TXA must not be used in a prophylactic manner, considering most of the patients and flaps being able to deal with the blood loss without treatment. Yet for patients with anaemia and a higher intraoperative or postoperative blood loss, TXA is 
Table 3 Complications TXA versus control group

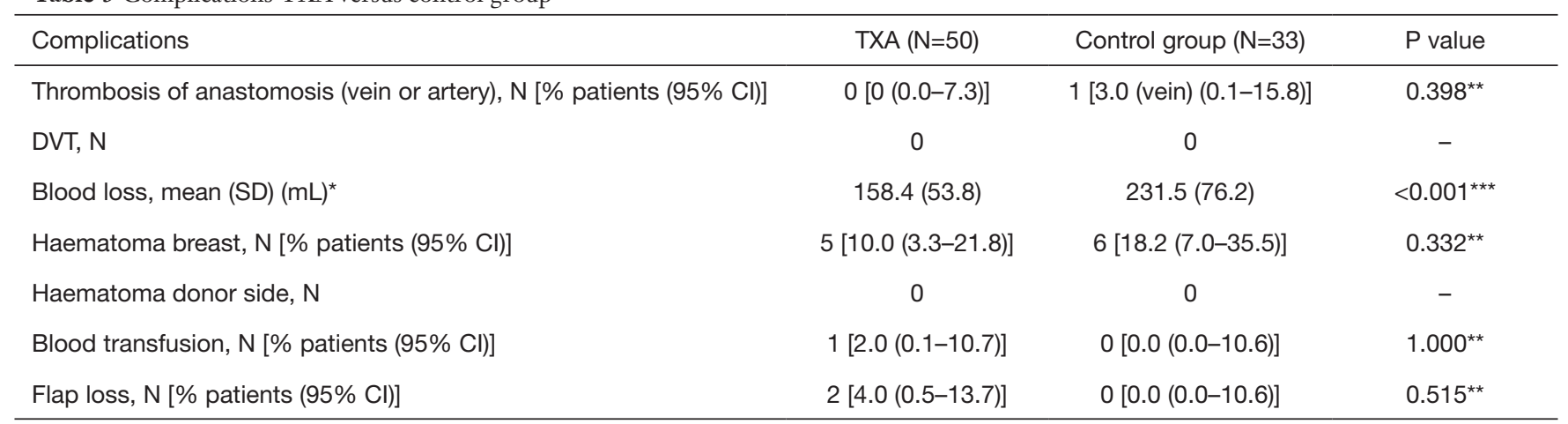

*, blood loss, sum of intraoperative blood loss and drainage in first 24 hours postop; ${ }^{\star \star}$, Fisher test; ${ }^{\star \star \star}$, ANOVA. TXA, tranexamic acid.

Table 4 Hematoma risk factors

\begin{tabular}{|c|c|c|c|c|c|}
\hline \multirow{2}{*}{ Risk factors } & \multirow{2}{*}{ Total number } & \multicolumn{4}{|c|}{ Haematoma } \\
\hline & & Number & $\%$ & Odds ratio* (95\% Cl) & $P$ value** \\
\hline TXA & 48 & 5 & 10.4 & & \\
\hline Control & 33 & 6 & 18.2 & & \\
\hline No & 61 & 9 & 14.8 & & \\
\hline Yes & 20 & 2 & 10.0 & & \\
\hline Neoadjuvant chemotherapy & & & & $2.37(0.41-13.58)$ & 0.333 \\
\hline No & 73 & 9 & 12.3 & & \\
\hline No & 75 & 9 & 12.0 & & \\
\hline Yes & 6 & 2 & 33.3 & & \\
\hline Flap type & & & & $5.80(1.52-22.15)$ & 0.010 \\
\hline DIEP & 45 & 3 & 6.7 & & \\
\hline PAP & 7 & 1 & 14.3 & & \\
\hline TMG & 18 & 6 & 33.3 & & \\
\hline S-GAP & 11 & 1 & 9.1 & & \\
\hline Age & & & & $10.00(1.18-84.76)$ & 0.035 \\
\hline
\end{tabular}

For two patients with multiple procedures the data from the procedure without use of TXA only were used. *, odds ratio was calculated as second versus first category, for flap type as TMG versus other types; ${ }^{\star \star}$, univariate logistic regression. DIEP, deep inferior epigastric perforator; PAP, profunda artery perforator; TMG, transverse myocutaneous gracilis; S-GAP, superior gluteal artery; TXA, tranexamic acid. 
considered a reasonable adjunct.

\section{Conclusions}

In this study, administration of TXA did not increase thrombosis in free tissue transfer and showed a reduced haematoma rate and significant reduction of blood loss. TXA is supposed to be safe and a reasonable adjunct for patients with anaemia and a higher intraoperative or postoperative blood loss.

\section{Acknowledgements}

None.

\section{Footnote}

Conflicts of Interest: The authors have no conflicts of interest to declare.

Ethical Statement: We hereby guarantee no ethical conflict in our study. The study is retrospective, no intervention was performed due to the study. The concept of the study was initiated in an audit of our institution to examine our practice.

\section{References}

1. Andree C, Langer S, Seidenstuecker K, et al. A single center prospective study of bilateral breast reconstruction with free abdominal flaps: a critical analyses of 144 patients. Med Sci Monit 2013;19:467-74.

2. Fosnot J, Fischer JP, Smartt JM Jr, et al. Does previous chest wall irradiation increase vascular complications in free autologous breast reconstruction? Plast Reconstr Surg 2011;127:496-504.

3. Barni S, Cabiddu M, Guarneri P, et al. The risk for anemia with targeted therapies for solid tumors. Oncologist 2012;17:715-24.

4. Weber RS, Jabbour N, Martin RC 2nd. Anemia and transfusions in patients undergoing surgery for cancer. Ann Surg Oncol 2008;15:34-45.

Cite this article as: Lardi AM, Dreier K, Junge K, Farhadi J. The use of tranexamic acid in microsurgery-is it safe? Gland Surg 2018;7(Suppl 1):S59-S63. doi: 10.21037/gs.2018.03.10
5. Nelson JA, Fischer JP, Grover R, et al. The impact of anemia on microsurgical breast reconstruction complications and outcomes. Microsurgery 2014;34:261-70.

6. Ausset S, Glassberg E, Nadler R, et al. Tranexamic acid as part of remote damage-control resuscitation in the prehospital setting: A critical appraisal of the medical literature and available alternatives. J Trauma Acute Care Surg 2015;78:S70-5.

7. Olsen JJ, Skov J, Ingerslev J, et al. Prevention of Bleeding in Orthognathic Surgery--A Systematic Review and Meta-Analysis of Randomized Controlled Trials. J Oral Maxillofac Surg 2016;74:139-50.

8. Li ZJ, Fu X, Xing D, et al. Is tranexamic acid effective and safe in spinal surgery? A meta-analysis of randomized controlled trials. Eur Spine J 2013;22:1950-7.

9. Wei $Z$, Liu M. The effectiveness and safety of tranexamic acid in total hip or knee arthroplasty: a meta-analysis of 2720 cases. Transfus Med 2015;25:151-62.

10. Ker K, Edwards P, Perel P, et al. Effect of tranexamic acid on surgical bleeding: systematic review and cumulative meta-analysis. BMJ 2012;344:e3054.

11. Ker K, Roberts I, Shakur H, et al. Antifibrinolytic drugs for acute traumatic injury. Cochrane Database Syst Rev 2015;5:CD004896.

12. Brinkman JN, Derks LH, Klimek M, et al. Perioperative fluid management and use of vasoactive and antithrombotic agents in free flap surgery: a literature review and clinical recommendations. J Reconstr Microsurg 2013;29:357-66.

13. Brands MT, van den Bosch SC, Dieleman FJ, et al. Prevention of thrombosis after microvascular tissue transfer in the head and neck. A review of the literature and the state of affairs in Dutch Head and Neck Cancer Centers. Int J Oral Maxillofac Surg 2010;39:101-6.

14. Enajat M, Aziz Mohammadi M, Debeij J, et al. Effect of acetylsalicylic acid on microvascular thrombosis in autologous breast reconstruction. J Reconstr Microsurg 2014;30:65-70.

15. Chen CM, Halvorson EG, Disa JJ, et al. Immediate postoperative complications in DIEP versus free/ muscle-sparing TRAM flaps. Plast Reconstr Surg 2007;120:1477-82. 